\title{
Quantum electron transfer processes induced by thermo-coherent state
}

\author{
SUMANA BANERJEE and GAUTAM GANGOPADHYAY* \\ S.N. Bose National Centre for Basic Sciences, JD Block, Sector-III, Salt Lake, Kolkata 700098 \\ e-mail: gautam@bose.res.in
}

MS received 16 June 2007; accepted 20 July 2007

\begin{abstract}
When the reactant surface is not in a thermal equilibrium, but in a thermo-coherent state we have derived the rate and discussed about the quantum features of the rate. In the limit of very low and very high temperature the expressions are derived analytically and compared with the case of thermal distribution. We have investigated the dependence of temperature on the rate due to displacement, distortion of the harmonic potential energy surfaces of the reactant and product manifold.
\end{abstract}

Keywords. Thermo-coherent state; electron transfer; quantum rate.

\section{Introduction}

The study of non-radiative processes in solids has provided a theoretical basis for understanding the electron transfer processes ${ }^{1-25}$ in condensed phases. For non-adiabatic interstate coupling between harmonic surfaces, Huang and Rhys, ${ }^{1}$ Kubo $^{2}$ and jointly by Kubo and Toyozawa ${ }^{3}$ had advanced the theory in the direction to understand the multiphoton processes in semiconductor. ${ }^{4}$ Marcus in 1956 from a reaction coordinate analysis gave a quantitative estimate of the rate of the outer sphere electron transfer in solution phase $^{5,6}$ corresponding to the terms of the thermal average Franck-Condon (FC) factor. ${ }^{16,20-22}$ This classical high temperature limiting expression of the non-adiabatic ET rate corresponds to the continuous dielectric medium which is given by,

$$
k=(2 \pi / \hbar) V^{2}\left(4 \pi k_{B} T \lambda\right)^{-1 / 2} \exp \left[-(\Delta G+\lambda)^{2} /\left(4 \lambda k_{B} T\right)\right]
$$

where $V$ is the non-adiabatic coupling constant and $\lambda$ is defined as the medium re-organization energy and $\Delta G$ is the free energy gap. Depending on the free energy change of the reaction with respect to medium re-organization energy factor $\lambda$, Marcus has classified the ET reactions into normal with, $-\Delta G<\lambda$, activationless when $-\Delta G=\lambda$ and the inverted regime where $-\Delta G>\lambda$, which still serves as the basic building block of the ET kinetics for donoracceptor system.

*For correspondence
Quantum effects have been studied related to electronic coupling between the two surfaces, ${ }^{16}$ namely, one electron two-centered exchange problem, ${ }^{7-10}$ many electron direct exchange problem was considered by Katz et $a l^{11}$ and by Silbey et $a l^{12}$ along with a bridge mediated processes in refs 13-15. Another major change in the work of ET processes was appeared to understand the quantum effects ${ }^{16-19}$ where the high frequency intramolecular vibrational modes along with the low frequency medium modes have been included.

In electron transfer theory quantum effect can also be important when the molecular and solvent reorganization are of quantum nature. Conditions for non-equilibrium reactant state are specially considered to account for the effect due to solvent dynamics $^{25}$ or for the ultrafast electron transfer processes. ${ }^{16}$ Vibrational coherence in electron transfer has been studied through the dynamically created wave packet by a femto second pulse in the excited state charge transfer complex of an electron donor-acceptor system between tetracyanoethylene and pyrene. ${ }^{26}$ Recently we have derived the expression of electron transfer rate ${ }^{27,28}$ for the solvent mode and intramolecular mode to incorporate the quantum nature of the potential surfaces of the reactants and products. As a non-thermal reactant state distribution, GlauberLachs state ${ }^{29,30}$ can be used which can be created in a harmonic oscillator mode in thermal equilibrium by a subsequent perturbation using an infra-red laser pulse. ${ }^{31}$ We have used the Franck-Condon factor ${ }^{23,24}$ to elucidate a detailed behaviour of the rate due to all the parameters of the potential surfaces in pres- 
ence of initial thermo-coherent state ${ }^{29,30}$ and thereby the quantum nature of the electron transfer rate.

In §2 we have formulated the rate of quantum eletron transfer processes for the reactant surface in thermo-coherent state instead of conventional thermal state. Franck-Condon factor is discussed for single mode and factorized two-mode displaced and distorted potential surfaces of the reactant and product. Section 3 is devoted to the result and discussion about the effect of potential surfaces on the rate due to thermo-coherent state. The conclusions are provides in $\S 4$.

\section{On the quantum effect in electron transfer}

The quantum effects in electron transfer in the low temperature tunneling regime and moderate to high temperature classical regime for the single and the two-mode cases have been studied extensively. ${ }^{16}$ In these studies the usual assumption is that the reactant state is in thermal equilibrium. However, an initial non-equlibrium reactant state is also considered in some cases. ${ }^{16,25,26}$ Here we have calculated the quantum rate due to thermo-coherent distribution of the reactant state in equilibrium.

For the single mode case the ET rate is given by the golden rule expression

$$
\begin{aligned}
& k=\frac{2 \pi|V|^{2}}{\hbar} \\
& \sum_{u} \sum_{v}\left|\left\langle\theta_{a v} \mid \theta_{b u}\right\rangle\right|^{2} \exp \left(-E_{a v} / k_{B} T\right) \delta\left(E_{b u}-E_{a v}\right) \\
& \sum_{v} \exp \left(-E_{a v} / k_{B} T\right)
\end{aligned}
$$

where the reactant state is in thermal equilibrium with $V$ is the coupling term between the reactant (a) and product (b) states. $\left|\left\langle\theta_{a v} \mid \theta_{b u}\right\rangle\right|^{2}$ is the FranckCondon factor between the reactant and product surfaces with $v$ and $u$ being the corresponding vibrational quantum number.

For a two-mode model the reactant and product states are harmonic oscillators where the product state oscillators are displaced and distorted from the reactant state. Let $\sum_{i} H_{i}^{g}$ and $\sum_{i} H_{i}^{e}$ be the Hamiltonian of the reactant and product states, respectively,

$$
H^{g}=E_{a}+\sum_{i=1}^{2} \frac{p_{i}^{g 2}}{2 M_{i}}+\frac{1}{2} M_{i}\left(\omega_{i}^{g}\right)^{2} x_{i}^{g 2}
$$

$$
H^{e}=E_{b}+\sum_{i=1}^{2} \frac{p_{i}^{e 2}}{2 M_{i}}+\frac{1}{2} M_{i}\left(\omega_{i}^{e}\right)^{2}\left(x_{i}^{e}-x_{i}^{0}\right)^{2} .
$$

$x_{i}^{g(2)}$ and $p_{i}^{g(e)}$ are the co-ordinates and momenta, respectively of mode $x_{i}^{0}$ is the shift of the equilibrium displacement in the product state corresponding to mode $i$. Here the dimensionless displacements are related to the real displacements, $x_{i}^{0}$ as

$$
Z_{i}=\sqrt{\frac{M_{i} \omega_{i}}{2 \hbar}} x_{i}^{0}
$$

and distortion factor of the $i$ th mode is

$$
r_{i}=\frac{1}{2} \ln \frac{\omega_{i}^{g}}{\omega_{i}^{e}}
$$

From the thermal average Golden rule expression for the two-mode case with frequencies $\omega_{1}^{\mathrm{g}}$ and $\omega_{2}^{\mathrm{g}}$ with dimensionless displacements $z_{1}$ and $z_{2}$ the rate is given by,

$$
\begin{aligned}
k= & \frac{|V|^{2}}{2 \pi \hbar} \sum_{u_{1}} \sum_{u_{2}} \sum_{v_{1}} \sum_{v_{2}} e^{-\left[\left(v_{1}+\frac{1}{2}\right) \hbar \omega_{1}^{g} / k_{B} T+\left[\left(v_{2}+\frac{1}{2}\right) \hbar \omega_{2}^{g} / k_{B} T\right]\right.} \\
& \left(1-e^{\hbar \omega_{1}^{g} / k_{B} T}\right)\left(1-e^{\hbar \omega_{2}^{g} / k_{B} T}\right) \\
& \left|\left\langle\theta_{a v_{1} v_{2}} \mid \theta_{b u_{1} u_{2}}\right\rangle\right|^{2} \\
& \delta\left(\hbar \omega_{00}+\hbar \omega_{2}^{g}\left(u_{2}-v_{2}\right)+\hbar \omega_{1}^{g}\left(u_{1}-v_{1}\right)\right) .
\end{aligned}
$$

Here $\hbar \omega_{00}$ is the value of the $0-0$ energy gap of the reactant and product surfaces. Considerable insight can be gained by assuming two classes of vibrational modes, a typical mode of the medium with frequency $\omega_{M}$ and a representative of the high frequency intramolecular modes with frequency $\omega_{c}$. Depending on the temperature of the reaction, the low frequency medium modes and high frequency intramolecular modes can be approximated nicely to give tangible analytical expressions for physical interpretation. ${ }^{16,24}$

\subsection{Quantum rate with Glauber-Lachs state}

Glauber-Lachs (GL) ${ }^{29-31}$ distribution of a harmonic oscillator interpolates between the Poissonian and thermal distributions. So it is sometimes called a thermo-coherent distribution. ${ }^{31}$ We consider the case when the reactant and product surfaces are harmonic 
oscillators which are displaced and distorted. When the reactant state is in a thermo-coherent state the rate depends on the two parameters of the distribution, namely, the thermal-average excitation number and the coherent-average excitation number of the vibrational states.

In the Glauber-Lachs state [GL], the population distribution in the $n$th state is given by

$$
P_{G L}(n)=\frac{N^{n}}{(1+N)^{n+1}} \exp \left(-\frac{S}{1+N}\right) L_{n}\left(-\frac{S}{N(N+1}\right)
$$

where $N$ and $S$ are the thermal-average and coherent-average excitation numbers of the states of the harmonic oscillator, respectively and

$$
L_{n}(x)=\sum_{i=0}^{n} \frac{(-x)^{i} n !}{(i !)^{2}(n-i) !}
$$

the Laguerre polynomial. The thermal average excitation number of frequency $\omega^{g}, N$ is given by

$$
N=\frac{1}{e^{\frac{\hbar \omega g}{k^{T}}}-1}
$$

When the coherent-average excitation number $S$ vanishes, the distribution becomes a thermal one, i.e.

$$
\lim _{S \rightarrow 0} P_{G L}(n)=\frac{N^{n}}{(1+N)^{n+1}}
$$

When the thermal-average excitation number $N$ vanishes, the distribution becomes Poissonian, i.e.

$$
\lim _{N \rightarrow 0} P_{G L}(n)=e^{-S} \frac{S^{n}}{n !}
$$

GL state can be obtained if an external Gaussian pulse of light excites a harmonic oscillator initially in a thermal distribution and then a thermalization takes place before considering the system as a candidate for electron transfer.

For the two-mode case the quantity of interest is the thermo-coherent average rate from the Fermi Golden rule expression

$$
k_{G L}=\frac{|V|^{2}}{2 \pi \hbar} \sum_{u_{1}} \sum_{v_{1}} \sum_{u_{2}} \sum_{v_{2}}\left|\left\langle\theta_{a v_{1}} \mid \theta_{b u_{1}}\right\rangle\right|^{2}
$$

$$
\begin{aligned}
& P_{G L}\left(N_{1}, S_{1}, \omega_{1}^{g}, v_{1}\right)\left|\left\langle\theta_{a v_{2}} \mid \theta_{b u_{2}}\right\rangle\right|^{2} \\
& P_{G L}\left(N_{2}, S_{2}, \omega_{2}^{g}, v_{2}\right) \delta\left(E_{b u_{1} u v_{2}}-E_{a v_{1}, v_{2}}\right) .
\end{aligned}
$$

For the numerical evaluation of $k_{G L}$ for single and two-mode cases we have written the delta-function in the integral representation. We have also considered a damping rate, $\gamma$ for the convergence of the integral whose magnitude does not affect the qualitative result through the numerical demonstration. For single-mode displaced oscillator case some analytical results in the limit of very high and very low temperature can be interesting for general understanding.

For a single mode displaced oscillator model with displacement in co-ordinate, $R_{0}$, with mass $M$ and distortion parameter $r=0$ i.e. frequency, $\omega^{g}=\omega^{e}=\omega_{0}$ and with $0-0$ energy gap, $E_{b}-E_{a}=\hbar \omega_{00}$, the rate is given by

$$
\begin{aligned}
k_{G L}= & \frac{|V|^{2}}{2 \pi \hbar^{2}} \int_{-\infty}^{\infty} d t e^{-i t\left(E_{b}-E_{a}\right) / \hbar} e^{-x^{2}} \\
& \sum_{u=0}^{\infty} \sum_{v=0}^{\infty}(-1)^{v-u} \exp \left[i t \omega_{0}(v-u)-\frac{S}{1+N}\right] \\
& \frac{N^{v}}{(1+N)^{v+1}} L_{v}\left(-\frac{S}{N(N+1)}\right) L_{v}^{(u-v)}\left(x^{2}\right) L_{u}^{(v-u)}\left(x^{2}\right) .
\end{aligned}
$$

Here the FC overlap factor is given by

$$
\left\langle\theta_{b u} \mid \theta_{a v}\right\rangle=e^{-x^{2} / 2}\left(\frac{v !}{u !}\right)^{1 / 2} x^{u-v} L_{v}^{u-v}\left(x^{2}\right),
$$

with

$$
x=\sqrt{\left(\frac{M \omega_{0}}{2 \hbar}\right) R_{0}}
$$

and

$$
L_{v}^{m}\left(x^{2}\right)=\sum_{i=\max (0,-m)}^{v} \frac{\left(-x^{2}\right)^{i}(m+v) !}{i !(v-i) !(m+i) !}
$$

where $m$ can be negative or positive integer including zero. By applying a special case of bi-linear generating function in the form ${ }^{27}$ 
$\sum_{0}^{\infty} A^{k} L_{k}^{(m-k)}(\chi) L_{m}^{(k-m)}(\chi)=A^{m} e^{-\chi A} L_{m}\left(2 \chi+\chi \frac{1+A^{2}}{A}\right)$,

we find that

$$
\begin{aligned}
k_{G L}= & \frac{|V|^{2}}{2 \pi \hbar^{2}} \int_{-\infty}^{\infty} d t e^{-i t \omega_{00}} \exp \left[-x^{2}\left(1-e^{-i t \omega_{0}}\right)\right] \\
& \sum_{v=0}^{\infty} \exp \left(-\frac{S}{1+N}\right) \frac{N^{v}}{(1+N)^{v+1}} \\
& L_{v}\left(-\frac{S}{N(N+1)}\right) L_{v}\left(2 x^{2}\left(1-\cos \omega_{0} t\right)\right) .
\end{aligned}
$$

Now making use of the formula ${ }^{32}$

$$
\begin{aligned}
& \sum_{n=0}^{\infty} \frac{n !}{\Gamma(\alpha+n+1)} L_{n}^{(\alpha)}(x) L_{n}^{(\alpha)}(y) z^{n} \\
& \quad=\frac{(x y z)^{-\alpha / 2}}{1-z} \exp \left(-z \frac{x+y}{1-z}\right) I_{\alpha}\left(2 \frac{\sqrt{x y z}}{1-z}\right)
\end{aligned}
$$

valid for $|z|<1$ where $I_{v}(z)$ denotes the modified Bessel function defined by

$$
I_{v}(x)=\sum_{k=0}^{\infty} \frac{(z / 2)^{v+2 k}}{k ! \Gamma(v+k+1)},
$$

we can simplify the integral in the following form:

$$
\begin{aligned}
k_{G L}= & \frac{|V|^{2}}{2 \pi \hbar^{2}} \int_{-\infty}^{\infty} d t e^{-i t \omega_{00}} \exp \left[-x^{2}\left(1-e^{-i t \omega_{0}}\right)\right] \\
& \exp \left[-i x^{2} \sin \left(\omega_{0} t\right)-2 x^{2}(1+2 N) \sin ^{2}\left(\omega_{0} t / 2\right)\right] \\
& I_{0}\left(4 i \sqrt{S} x \sin \left(\omega_{0} t / 2\right)\right) .
\end{aligned}
$$

To calculate the rate at very low temperature limit from (10) we have

$$
\begin{aligned}
k_{G L}= & \frac{|V|^{2}}{2 \pi \hbar} e^{-x^{2}-S} \sum_{u=0}^{\infty} \sum_{v=0}^{\infty}(-1)^{v-u} \frac{S^{v}}{v !} L_{v}^{(u-v)}\left(x^{2}\right) \\
& L_{u}^{(v-u)}\left(x^{2}\right) \delta\left(\hbar \omega_{00}+\hbar u \omega_{0}-\hbar v \omega_{0}\right),
\end{aligned}
$$

where we have considered the limiting value of

$$
\frac{N^{v}}{(1+N)^{v+1}} L_{v}\left(-\frac{-S}{N^{2}+N}\right)=\sum_{k=0}^{v} \frac{S^{k} N^{v-k}}{k !} \approx \frac{S^{v}}{v !} .
$$

The final expression of rate after simplifying the deltafunction is given by,

$$
\begin{aligned}
k_{G L}= & \frac{|V|^{2}}{2 \pi \hbar\left(\hbar \omega_{0}\right)} \\
& e^{-x^{2}-S} \sum_{u=0}^{u_{\max }} \frac{(-S)^{p}}{p !}(-1)^{u} L_{p}^{(u-p)}\left(x^{2}\right) L_{u}^{(p-u)}\left(x^{2}\right)
\end{aligned}
$$

where

$$
p=\frac{\mid \omega_{00}+u \omega_{0}}{\omega_{0}} \quad \text { and } \quad u_{\max }=\frac{\left|\omega_{00}\right|}{\omega_{0}} .
$$

In the limit $S$ tends to zero and $p$ becomes zero, the rate is given by

$$
k_{G L}=\frac{|V|^{2}}{2 \pi \hbar \omega_{0}} e^{-x^{2}} \frac{\left(x^{2}\right)^{u_{\max }}}{u_{\max } !} .
$$

This is precisely the nuclear tunneling rate $^{16}$ for thermal distribution at zero temperature which is clarified by Jortner from the Huang-Rhys formula. ${ }^{1,16}$

To calculate the time-integral we assume short time approximation of (17) which is valid at moderately high temperature limit. For $S=0$, the steepest descent approximation of the thermal-averaged bandshape function works well. Therefore, we consider the Gaussian approximation for the first part, i.e. the $S=0$ part of the bandshape function. For the second term, i.e. $I_{0}(z)$ term, we shall consider shorttime approximation. The exponential term of the integrand can be considered in the form

$$
f_{a b}=-i t \omega_{00}-i x^{2} \omega_{0} t-x^{2} \omega_{0}^{2} \frac{t^{2}}{2} C,
$$

where

$$
C=1+2 N,
$$

with $C=\operatorname{coth}\left(\hbar \omega_{0} / 2 k_{B} T\right)$. Therefore, we have,

$$
f_{a b}^{\prime}(t)=-i \omega_{00}-i x^{2} \omega_{0}-x^{2} \omega_{0}^{2} t C,
$$


and

$$
f_{a b}^{\prime \prime}(t)=-x^{2} \omega_{0}^{2} C
$$

Now for $f_{a b}^{\prime}\left(t^{*}\right)=0$ one obtains

$$
t^{*}=\frac{-i \omega_{00}-i x^{2} \omega_{0}}{x^{2} \omega_{0}^{2} C}
$$

and writing

$$
f_{a b}(t)=f_{a b}\left(t^{*}\right)+\frac{1}{2} f_{a b}^{\prime \prime}\left(t^{*}\right)\left(t-t^{*}\right)^{2}
$$

one finds

$$
f_{a b}\left(t^{*}\right)=\frac{-\left\{\left(\omega_{00}\right)+x^{2} \omega_{0}\right\}^{2}}{2 x^{2} \omega_{0}^{2} C}
$$

Thus the rate can be written as

$$
k_{G L}=\frac{\exp \left[f_{a b}\left(t^{*}\right)\right]}{\left[-2 \pi f_{a b}^{\prime \prime}\left(t^{*}\right)\right]^{1 / 2}} .
$$

Now, in the $I_{0}(\mathrm{z})$ term of (17), we assume the shorttime limit and set $t=t^{*}$, the rate, $k_{G L}$ can be given by

$$
\begin{aligned}
k_{G L}= & {\left[\frac{2 \pi}{\hbar^{2}} \lambda k_{B} T\right]^{-1 / 2} \exp \left[-\frac{\left(\hbar \omega_{00}+\lambda\right)^{2}}{2 \lambda k_{B} T}\right] } \\
& I_{0}\left(2 \sqrt{S} x \frac{\hbar \omega_{0}\left(\hbar \omega_{00}+\lambda\right)}{\lambda k_{B} T}\right)
\end{aligned}
$$

where

$$
\lambda=\hbar x^{2} \omega_{0} \text { with } x=\sqrt{\frac{M \omega_{0}}{2 \hbar}} R_{0} \text { and } N=\frac{1}{e^{\frac{\hbar \omega_{0}}{k_{B}}}-1} .
$$

In the high temperature limit it is assumed that $\left.\cot h\left(\hbar \omega_{0} / 2 k_{B} T\right) \approx k_{B} T / \hbar \omega_{0}\right)$. The rate has a non-trivial dependence on $S$. For $S=0$, the rate reduces to the Marcus one corresponding to the thermal distribution. However, when $S \neq 0$, the activationless reaction condition, i.e. when $\hbar \omega_{00}+\lambda=0$ the reaction rate is the same as in thermal distribution case. But in the normal or inverted regime, i.e. for the case of $\hbar \omega_{00}<\lambda$ or, $\hbar \omega_{00}>\lambda$ the rate is affected largely by $S$. In short, $S$ can switch an electron transfer reaction from normal to the inverted regime and vice versa.
We have considered the $G-L$ state as an initial quantum distribution to show the effect on the rate of the electron transfer reaction. When the vibrational mode of the reactant state is driven by an external IR-laser field as well as the vibrational mode is damped, a steady state is reached which is the Glauber-Lachs state. This steady state appears during the damping time of the mode. The electron transfer reaction should also start when the $G-L$ state is achieved in the reactant mode. So the preparation of $G-L$ state and electron transfer reaction should be really separated in time unless the thermalization process is fast enough. However, the time scale of thermalization in presence of external laser depends on many factors, e.g. strength of the laser field, damping rate of the vibrational mode, frequency of the mode and temperature of the bath, etc. These dependence can be understood from the derivation of $G L$ state as a steady state distribution by Filipowicz. ${ }^{31}$

\section{Franck-Condon factor}

Before putting the numerical result in proper perspective, in this section we have briefly discussed how the quantum effect arises due the nature of interactions in the reactant and product manifolds. The quantum effect on the electron transfer rate heavily depends on the parameters of the potential surfaces. We have considered the effect through the FranckCondon overlap integral between the two adiabatic surfaces $^{24}$ for the multimode displaced distorted and Duschinsky rotated oscillators in the number state representation.

When the reactant and product states are oscillators with $H_{i}^{q}$ and $H_{i}^{e}$ be the Hamiltonian of the reactant and product states of mode $i$, respectively,

$$
\begin{aligned}
& H_{i}^{g}=\frac{p_{i}^{g 2}}{2}+\frac{1}{2}\left(\omega_{i}^{g}\right)^{2} x_{i}^{g 2}, \\
& H_{i}^{e}=\frac{p_{i}^{e 2}}{2}+\frac{1}{2}\left(\omega_{i}^{e}\right)^{2}\left(x_{i}^{e}-x_{i}^{0}\right)^{2} .
\end{aligned}
$$

$x_{i}^{g(e)}$ and $p_{i}^{g(e)}$ are the mass weighted co-ordinates and momenta, respectively of mode $x_{i}^{0}$ is the shift of the equilibrium displacement in the product state corresponding to mode $i$. The co-ordinates of the product state oscillators are

$$
x^{e}=W x^{g}+x^{0},
$$


where

$$
W_{i j}=\sqrt{\frac{\omega_{i}^{g}}{\omega_{j}^{e}}} R_{i j} .
$$

Here $R$ is the Duschinsky rotation matrix corresponding to mixing between the modes. In absence of Duschinsky rotation, the matrix $W$ is diagonal and the rotation matrix, $R$ is an unit matrix i.e. $R=1$. The evaluation of FC factor involves the transformation of co-ordinates from $x^{g}$ to $x^{e}$ along with the conjugate momenta $p^{e}$ of the product oscillator.

However, for our numerical studies of single and factorized two-mode ET process we consider the single mode displaced-distorted oscillator FC overlap integral, $\left\langle\theta_{n}^{g} \mid \theta_{k}^{e}\right\rangle$ as

$$
\begin{aligned}
& \left\langle\theta_{n}^{g} \mid \theta_{k}^{e}\right\rangle=\frac{1}{\sqrt{\cosh (r)}} \exp \left[-\frac{z^{2} e^{-r}}{2 \cosh (r)}\right] \\
& \sqrt{n ! k !}\left(-e^{r}\right)^{n}(-1)^{k}\left(\frac{1}{\cosh (r)}\right)^{n+k} \\
& \sum_{j=0}^{n / 2} \sum_{k_{1}=0}^{k / 2} \sum_{l=0}^{\min \left(n-2 j, k-2 k_{1}\right)} z^{\left(k+n-2 j-2 k_{1}-2 l\right)} \\
& \left(\frac{\sinh (r) \cosh (r)}{2 e^{-2 r}}\right)^{j}\left(-\frac{\sinh (r) \cosh (r)}{2}\right)^{k_{1}} \\
& \left(-\frac{\cosh (r)}{e^{-r}}\right)^{l} \frac{1}{j ! k 1 ! l !(n-2 j-l) !\left(k-2 k_{1}-l\right)} \text {. }
\end{aligned}
$$

Here the distortion parameter $r=1 / 2 \ln \left(\omega^{g} / \omega^{e}\right)$ and the dimensionless displacement parameter, $z=x_{0} \sqrt{\omega^{g} / 2 \hbar}$. In the upper limits of the summations, $n / 2$ and $k / 2$ are to be taken up to the highest integer values for the odd values of $n$ and $k$. Note that the F.C. overlap integral depends on $r, x_{0}$ and also on the explicit value of the one of the frequencies, $\omega^{g(e)}$.

\section{Results and discussion}

To explore the quantum effect of the electron transfer rate we have numerically investigated, how the rate of electron transfer in a molecular system is influenced by the temperature, electronic energy gap and other parameters of the potential surfaces, like displacement $z$, distortion $r$. For a two-mode case we have studied the effect of a parameter called $R$ which is the ratio of vibrational frequencies of the reactant state. For the single mode case it can be assumed that the mode physically corresponds to the solvent and for the two-mode case the other mode can be of molecular origin or corresponding to the solvent mode.

We have shown the variation of rate, $k(T)$ (in arbitrary unit) against energy gap, $\Delta=\hbar \omega_{00}$ and secondly the variation of scaled rate, $k(T) / k(0)$ against temperature where $k(0)$ is the rate at zero temperature. We have considered single mode displaced-distorted oscillator system as a model and also a two mode displaced distorted oscillator molecular system to perform the following investigations. In all cases we have considered a factor of convergence $\gamma=0.5 \omega_{1}^{g}$ by writing the delta function in terms of a Lorenztian in the expression of rate with width $\gamma$. We have considered that all quantities have been scaled with respect to the vibrational frequency of the first mode of the reactant surface, $\hbar \omega_{1}^{g}$ for all cases. Temperature is expressed in the units of $\hbar \omega_{1}^{g} / \mathrm{kB}$ for the single mode and two-mode cases. For the two-mode cases unless otherwise specified we have taken the modes with frequencies $\omega_{2}^{g}=0.5 \omega_{1}^{g}$. In both the modes we have
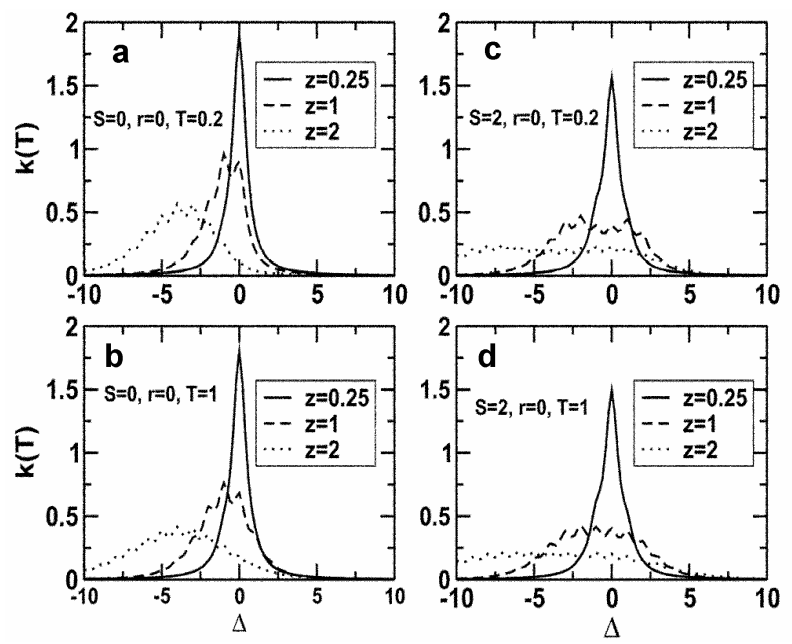

Figure 1. For single-mode case, the rate of electron transfer, $k(T)$ (in arbitrary unit) is plotted against energy gap, $\Delta$. For $r=0, T=0.2$ in (a) $S=0$ and in (b) $S=2$ and similarly for $r=0, T=1$ in (c) $S=0$ and in (d) $S=2$, the curves are given with increase in displacement parameter, $z$ from 0.25 to 2 . Like the temperature effect, with increase in $S$ the rate profile broadens. 
taken displacements, $z_{1}=z_{2}=z$, distortion factors $r_{1}=r=r_{2}$, average coherent excitations, $S_{1}=S_{2}=S$ and ground state temperatures, $T_{1}=T_{2}=T$.

First, we consider the variation of rate against the energy gap, $\Delta$ for single mode system. In figure 1(a-d) we plot the rate against electronic energy gap $\Delta$ to observe the effect of displacement parameter $z$ at two different reactant state temperature $T=0.2$ and $T=1$ in units of $\hbar \omega_{1}^{q} / k_{B}$ each with two different values of $S$. At each temperature two different $S$ values are
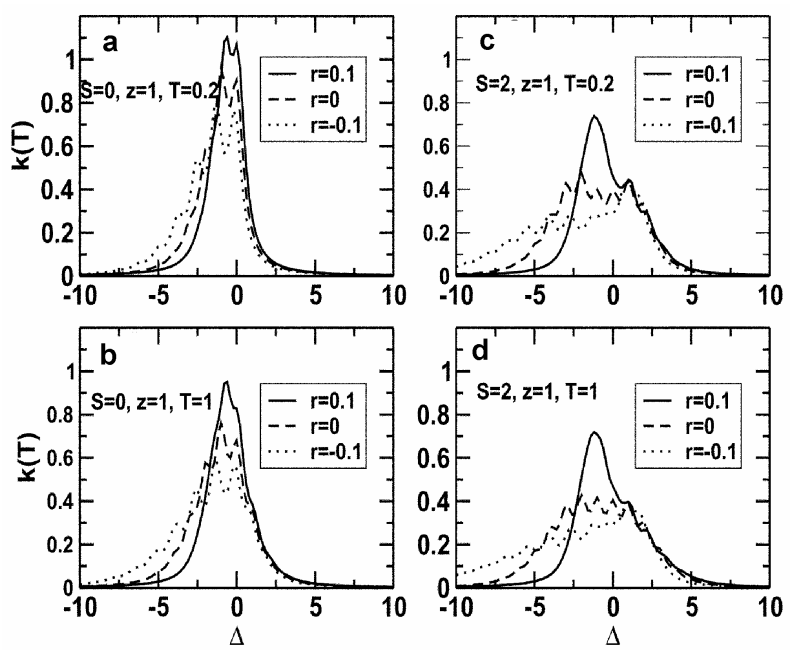

Figure 2. For single-mode case, the rate of electron transfer, $k(T)$ (in arbitrary unit) is plotted against energy gap, $\Delta$. For $z=1, T=0.2$ in (a) $S=0$ and in (b) $S=2$ and similarly for $z=1, T=1$ in (c) $S=0$ and in (d) $S=2$, the curves are given with decrease in distortion parameter, $r$ from $0 \cdot 1$ to $-0 \cdot 1$. Like the temperature effect, with increase in $S$ the rate profile broadens.
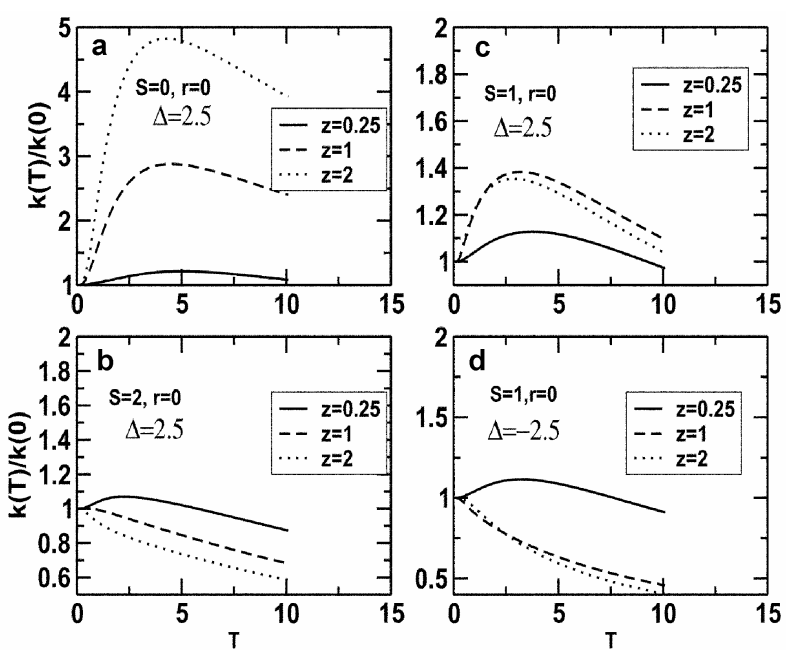

Figure 3. For single-mode case, plots of the scaled rate, $k(T) / k(0)$ of electron transfer against reactant state temperature $T$ are provided to show the effect of displacement. We have taken $r=0, \Delta=2.5$ in (a) $S=0$ and in (b) $S=2.0$ and similarly for $r=0, S=1.0$ in (c) $\Delta=2.5$ and in $(\mathbf{d}) \Delta=-2 \cdot 5$.
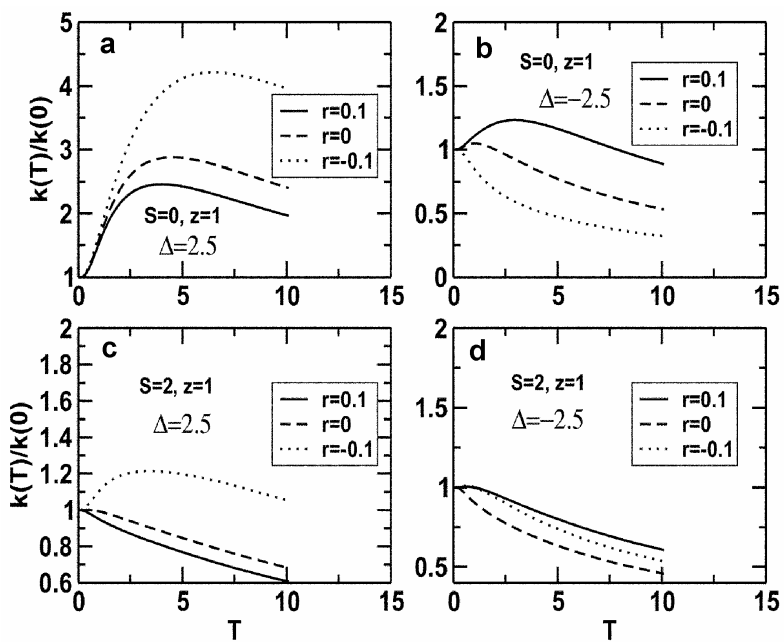

Figure 4. For single-mode case, plots of the scaled rate, $k(T) / k(0)$ of electron transfer against reactant state temperature $T$ are provided to show the effect of distortion. We have taken $z=1, \Delta=2.5$ in (a) $S=0$ and in (c) $S=2.0$ and similarly for $z=1, \Delta=-2.5$ in (b) $S=0$ and in $(D) S=2$.
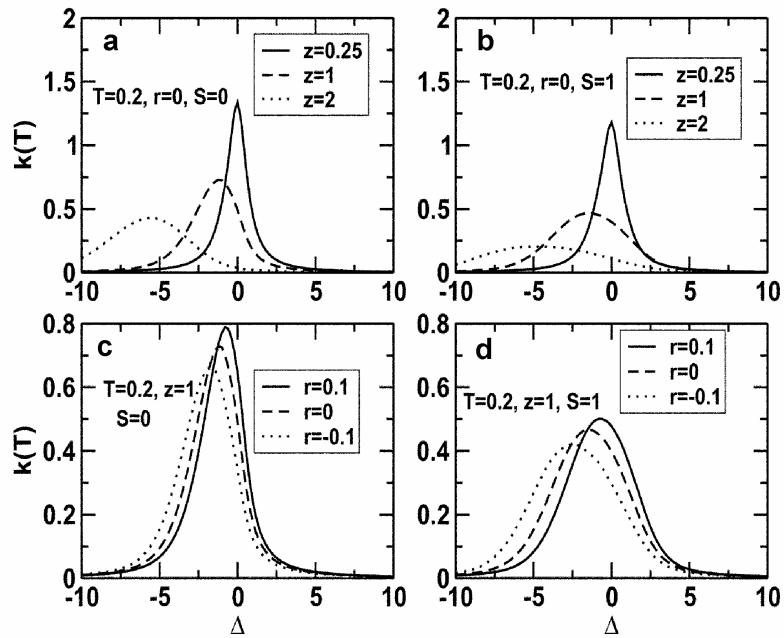

Figure 5. For two-mode case the rate of electron transfer is plotted against $\Delta$ to show the variation of $z=z_{1}=z_{2}$ with $r_{1}=r_{2}=r=0, T=0 \cdot 2$ at $S=0$ in (a) and at $S=1$ in (b). Similarly in (c) and (d) variation of rate profile is shown with distortion at a fixed value of $z=z_{1}=z_{2}=1$ and $T=T_{1}=T_{2}=0.2$ in (c) for $S=0$ and in (d) for $S=1$. 
chosen, for example, for $T=0 \cdot 2$, in (A) $S=0$ and in (B) $S=2$. In figure $1(\mathrm{a}-\mathrm{d})$ we vary $z$, keeping no distortion, i.e. $r=0$. It is seen that the maximum in the rate appears approximately at $-z^{2}$ in accordance with the high temperature classical rate of Gaussian expression. For high temperature case the role of reorganization energies in the energy-gap law is physically clear and the corresponding temperature dependence on the rate. ${ }^{24}$ As $z$ increases, the maximum shifts towards the left and the rate also decreases. It is well known that with increase in temperature the rate profile broadens with $\Delta$. With increase in $S$ also we find the rate profile broadens and it broadens more than temperature effect. At higher $z$-value the peak passes through a minimum which is typical for a Glauber-Lachs distribution case. This corresponds to the fact that in the activationless situation the rate decreases.

Similarly in figure 2(a-d), in each figure we show the rate profile with $\Delta$ with different values of distortion factor, $r$ for a fixed value of $S$ and temperature $T$. The effect of $r$ appears through the asymmetry of the curves with more signature of vibrational levels in the potential surfaces than in case of figure $1(\mathrm{a}-\mathrm{d})$. The case corresponding to $r=0$ means the frequencies of oscillators in the ground and excited states are the same. A systematic variation of $r$ shows broadening of the rate profile with increase in $S$ at a fixed temperature. The temperature effect is as usual broadening of the rate profile.
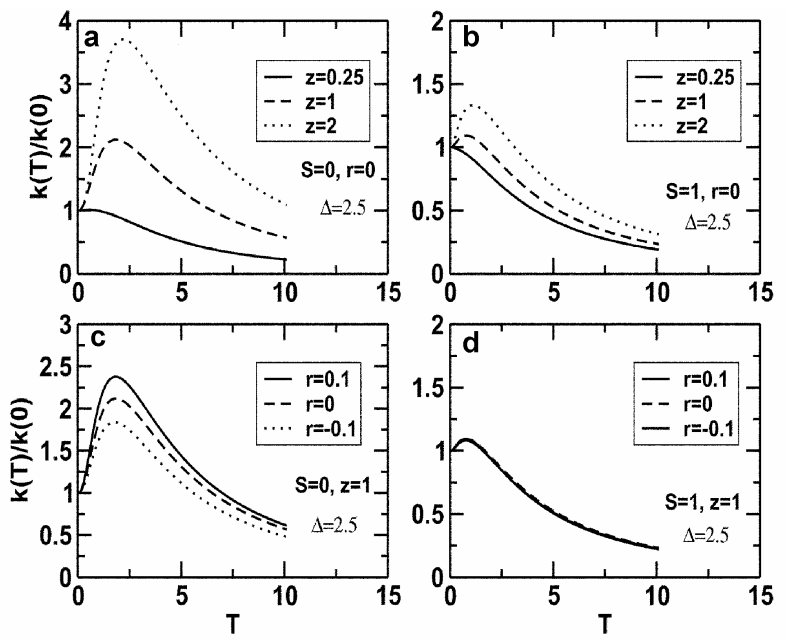

Figure 6. For two-mode case the relative rate, $k(T) / k(0)$ is plotted against Temperature, $T=T_{1}=T_{2}$ to show the variation with $\mathrm{z}=z_{1}=z_{2}$ in (a) with $S=0$ and (b) with $S=1$ and the variation of $r=r_{1}=r_{2}$ in (c) $S=0$ and (d) $S=1$, for a fixed value of $\Delta=2 \cdot 5$.
In figures 3 and 4 we have shown the variation of scaled rate, $k(T) / k(0)$ against temperature for single mode case to analyse the effect of various parameters of the potential surfaces. In figure 3 , the displacement is varied from $z=0.25$ to $z=2.0$ for a fixed value of distortion parameter, $r=0$. It is seen that as the negative value of $\Delta$ increases from $-2 \cdot 5 \omega^{g}$ to $2 \cdot 5 \omega^{g}$, the rate becomes higher. With increase in $S$, say, from $S=0$ (figure 3a), through $S=1.0$ for figure $3 b$ to $S=2.0$ for figure 3c, the rate decreases. If we compare figures $3 \mathrm{c}$ and $\mathrm{d}$, the trend in temperature dependence with the displacement are opposite for positive and negative $\Delta$ which is apparently follow from the rate profile in figure 1 . Similarly in figures $4(a-d)$ we have shown the dependence of temperature on the rate with some fixed values of distortion parameter, $r$. Here if we compare figure 4(b) and (c), with change in $\Delta$ the trend is the opposite. Similar effect is observed in figure 4(d). It can be understood qualitatively from figure 2 where it is found that the effect of increase in $S$ in negative and positive $\Delta$ are of opposite trend for the different curves corresponding to the various values of $r$.

In figure 5 we have shown the rate profiles for two-mode system with $\Delta$ for some fixed values of displacement in figure $5(\mathrm{a}-\mathrm{b})$ and distortion in figure 5(c-d). For the two-mode case the vibrational level
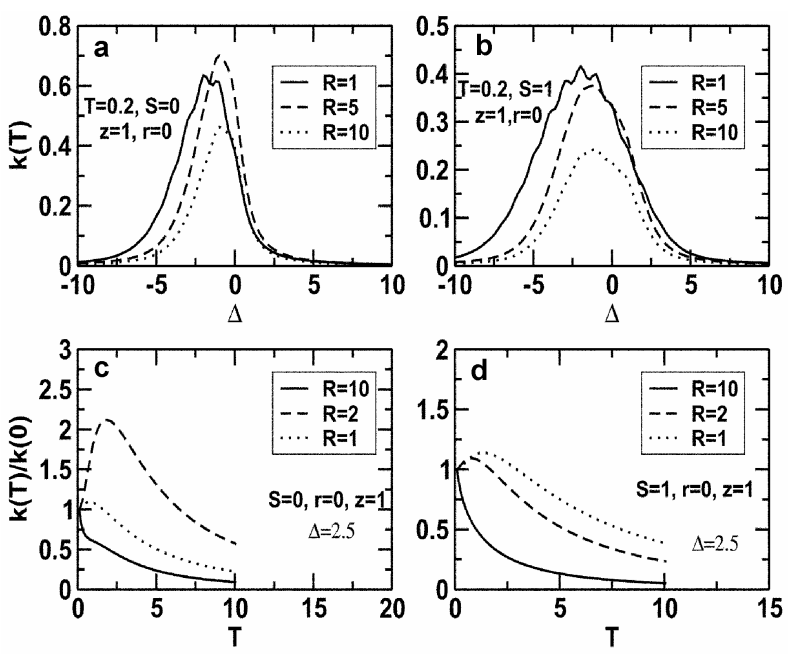

Figure 7. For two-mode case, in (a) and (b) the rate of electron transfer $k(T)$ is plotted against $\Delta$ and in (c) and (d) the relative rate, $k(T) / k(0)$ is plotted against Temperature, $T=T_{1}=T_{2}$ to show the variation with the ratio of frequencies of the two modes in the reactant state, $R=\omega_{1}^{g}$ $/ \omega_{2}^{g}$ and $z=z_{1}=z_{2}$. In the first cases, (a) and (b) with increase in $R$ the rate profile broadens and in the second case in (c) and (d), the quantum nature decreases. 
structures are absent in the rate profile. The displacement and distortion dependence are like single mode cases. With increase in $S$ the rate profiles are broadened where we have considered $S=S_{1}=S_{2}$. For the two mode case the rate profiles are more symmetric than the single mode case.

In figure $6 \mathrm{a}-\mathrm{b}$, we have shown the trend in temperature dependence with the displacement for different $S$. Here also we consider $S=S_{1}=S_{2}$. For both figure $6 \mathrm{a}-\mathrm{b}$ and c-d pairs, with increase in $S$ the classical nature of temperature depenedence increases.

In figure $7 \mathrm{a}-\mathrm{b}$ we have shown the plots of rate with $\Delta$ for two-mode system where the various curves in a plot are different by a parameter, $R$ which is the ratio of the frequencies of the oscillators of the two modes in the reactant state. It is apparent that with increase in $R$ the vibrational overlap becomes wider or in other words more de-phasing is introduced in the system during the transition from the reactant to the product states. We find that with increase in $S$, the rate profile is broadened and the height decreases as in the single mode case. Similarly, in figure $7 \mathrm{c}-\mathrm{d}$, the variation of scaled rate, $k(T) / k(0)$ is plotted against temperature. Here also we see that with increase in $S$ the quantum behaviour of the rate decreases.

\section{Conclusion}

While the quantum theory of electron transfer problems for single-mode and two-mode cases are studied for a long time from the low temperature tunneling regime to moderate to high temperature classical regime, the quantum effects are not ex-haustively investigated with its full complexities of the parameters of the system. In most of the cases it is assumed that the reactant is in thermal equilibrium. In this context we have explicitly calculated the quantum effect due to thermo-coherent reactant state distribution instead of thermal distribution for single and two mode cases and have shown the low and high temperature limits and compared with the thermal state case. The other quantum effect that can come from the nature of the potential surfaces and consequently through the Franck-Condon factor where the effect of displacement and distortion are considered in a systematic way.

Another feature of quantum electron transfer theory is about the various interesting other quantum state of the reactant surface which may be created by some pulsed laser source or by some special property of the quantum nature of the solvent. Here we have considered a Glauber-Lachs state, which is an intermediate between the coherent and thermal state for single mode case. We have provided analytical expression for the rate with GL state and have shown its limiting behaviour in the usual thermal rates at low and high temperature regime. GL state can be generated if an external Gaussian pulse of light excites a harmonic oscillator initially in a thermal distribution. However, a time scale separation is necessary between the preparation of thermalized steady state distribution of GL state and the consequent electron transfer from GL state in the scheme adopted here. It is shown that in the GL state with the activationless reaction condition, in presence of extra coherent vibrational excitations in addition to thermal vibrational excitation at a moderate temperature, the ET rate is not affected. Although the quantum effect is predominant in low temperature regime for thermal reactant state, in the thermocoherent state even at low temperature but with higher average coherent excitation the rate behaves classically.

\section{References}

1. Huang K and Rhys A 1950 Proc. Roy. Soc. Lond. A204 406

2. Kubo R 1952 Phys. Rev. 86929

3. Kubo R and Toyozawa Y 1955 Prog. Theo. Phys. 13 160

4. Seitz F 1951 Rev. Mod. Phys. 23328

5. Marcus R A 1956 J. Chem. Phys. 24 966, 979

6. Marcus R A 1993 Rev. Mod. Phys. 65599

7. Levich V G 1966 Adv. Electrochem. Electrochem. Eng. 4249

8. Dogonadze R R, Kuznetsov E M and Levich V G 1968 Electrochim. Acta 131025

9. Dogonadze R R and Kornyshev A A 1972 Phys. Status Solidi 53439

10. Dogonadze R R, Kuznetsov E M and Vorotyntsev M A 1972 Phys. Status Solidi 54125

11. Katz J L, Rice S A, Choi S I and Jortner J 1963 J. Chem. Phys. 391683

12. Silbey R, Jortner J, Rice S A and Vala M T $1965 \mathrm{~J}$. Chem. Phys. 42 733, Silbey R, Jortner J, Rice S A and Vala M T 1965 J. Chem. Phys. 432525

13. George M and Griffith J S 1959 in The enzymes I (New York: Academic) p 347

14. Helpern J and Orgel L E 1960 Disc. Farad. Soc. 29 32

15. McConnell H M 1961 J. Chem. Phys. 35508

16. Bixon M and Jortner J 1999 Adv. Chem. Phys. 106 35. Special issue on electron transfer from isolated molecules to biomolecules, Part-I (eds) J Jortner and $\mathrm{M}$ Bixon) see references therein 
17. Kestner N R, Logan R and Jortner J 1974 J. Phys. Chem. 782148

18. Ulstrup J and Jortner J 1975 J. Chem. Phys. 634358

19. Jortner J 1976 J. Chem. Phys. 644860

20. Tang J, Lee M T and Lin S H 2003 J. Chem. Phys. 1197188

21. Markham J J 1959 Rev. Mod. Phys. 31956

22. Yan Y J and Mukamel S 1986 J. Chem. Phys. 85 5908

23. Banerjee S and Gangopadhyay G 2005 J. Chem. Phys. 123114304

24. Banerjee S and Gangopadhyay G 2007 J. Chem. Phys. 126034102
25. Bixon M and Jortner J 1989 Chem. Phys. Letts. 159 17

26. Wynne K, Reid G D and Hoschstrasser 1999 Adv. Chem. Phys. 107263 (ed.) M. Bixon and J Jortner

27. Gangopadhyay G 1999 J. Phys. A32 L433; Gangopadhyay G 1998 J. Phys. A31 L771

28. Srivastava H M and Gangopadhyay G 2004 Russ. J. Math. Phys. 11359

29. Glauber R J 1963 Phys. Rev. 1312766

30. Lachs G 1965 Phys. Rev. 1381012

31. Filipowicz P 1986 J. Phys. A19 3785

32. Gradshteyn I S and Ryzhik I M 1980 Table of integrals, series and products (NY: Academic) 